\title{
Dynamics of a host-parasite model connected with immigration
}

\author{
Ozlem Ak Gumus ${ }^{1}$, Figen Kangalgil ${ }^{2}$ \\ ${ }^{1}$ Adiyaman University, Faculty of Arts and Sciences, Department of Mathematics, 02040, Adiyaman, Turkey. \\ ${ }^{2}$ Cumhuriyet University, Faculty of Science, Department of Mathematics, 58140, Sivas, Turkey.
}

Received: 9 February 2017, Accepted: 8 September 2017

Published online: 17 October 2017.

\begin{abstract}
The growth of the populations depending on interspecific interactions may exhibit drastic changes. The stability of populations change not only interspecific interactions but also under external effects such as immigration effect. Thus, population models can show complex dynamics. Immigration effect often simplify the population dynamics; and tends to supress chaotic behavior. This situation which can allow the local stability analysis of population is important for control of a two species interacting system. In this paper, we investigated dynamics of a host-parasite model with the immigration parameter added to the host population under the constant searching efficiency. We conclude that immigration parameter produces certain interesting results.
\end{abstract}

Keywords: Host-parasite model, stability analysis, equilibrium point, discrete dynamical systems, Misra-Mitra model.

\section{Introduction}

The population models consisting interspecific interactions are formed by discrete-time model and continuous-time models. Discrete-time models are more appropriate than continuous-time models in point of rich set of patterns [2]. One of the earliest applications of discrete-time models including host-parasite interaction was formulated by Leslie and Gower in 1960. The classical host-parasite model is the Nicholson-Bailey model, except that the parasite does not necesserily kill the host $[1,7,8]$. This model is based on the following assumptions;

$f\left(H_{t}, P_{t}\right)$ :fraction of hosts not parasitized,

$H_{t}$ :density of host species in generation $t$,

$P_{t}$ :density of parasitoid species in generation $t$

$r$ :number of eggs laid by a host that survive through the larvae, pupae and adult stages,

$e$ :number of eggs laid by parasite on a single host that survive through larvae, pupae and adult stages.

The Nicholson-Bailey model is given as follows.

$$
\begin{aligned}
H_{t+1} & =r H_{t} f\left(H_{t}, P_{t}\right) \\
P_{t+1} & =e H_{t}\left(1-f\left(H_{t}, P_{t}\right)\right)
\end{aligned}
$$

where, $r$ and $e$ are positive parameters.

It assume in this model that searching efficiency of parasitoids are limited but eggs aren't limited. If the searching 
efficiency of parasitoid has small values, parasitoid can vanish. The eggs of parasite which stay alive are transferred to the next generations of parasitoids. A host is parasitized for once. Interference-free hosts increase their own progeny.

The number of encounters of the parasitoid with a host according to the law of mass action is defined $c H_{t} P_{t}$. Here, the constant $c$ is defined as the searching efficiency. Nicholson-Bailey model assumes that the number of encounters are calculated according to the Poisson distribution such that $p(n)=e^{-\sigma} \sigma^{n} / n$ !, where $n$ is the number of encounters and $\sigma$ is the avarege number of encounters per host in one generation. If there is no encounter, the fraction of hosts that are not parasitized is $p(0)=e^{-\sigma} \sigma^{0} / 0 !=e^{-\sigma}$, where $\sigma=$ encounters $/ H_{t}=c P_{t}$. Then $f\left(H_{t}, P_{t}\right)=e^{-c P_{t}}$. Accordingly, the model is given as

$$
\begin{aligned}
H_{t+1} & =r H_{t} e^{-c P_{t}} \\
P_{t+1} & =e H_{t}\left(1-e^{-c P_{t}}\right) .
\end{aligned}
$$

If constant reproductive rate of host has small values, the host can vanish. Then, the positive equilibrium point of the models consisting density-dependent factor instead of a constant reproductive rate of the host can be locally stable [4,9]. Even so, there are many reasons affecting the number of species such as immigration, human activities, and interaction of among species. If the density-dependent factor $g\left(H_{t}\right)$ is added instead of a constant reproductive rate $r$ of in the host equation, the following more realistic approach is written as

$$
\begin{aligned}
H_{t+1} & =g\left(H_{t}\right) H_{t} e^{-c P_{t}} \\
P_{t+1} & =e H_{t}\left(1-e^{-c P_{t}}\right) .
\end{aligned}
$$

The the some forms of density-dependent factor (interspecific interactions) $g\left(H_{t}\right)$ has been studied in $[4,6,9,12]$. Misra and Mitra (2006) examined dynamics of discrete single-species population for different values of the parameter $R$ and $b$ which depend on Hassel growth function expessed with $\frac{R}{\left(1+H_{t}\right)^{b}}$. The results clearly show that the population display period-doubling phenomenon for $b>2$, and bifurcation occur with increasing $b$. As the parameters increase, the behavior of population changes from stability to unstable. Also, host-parasite system is given a brief analysis [6]. Ufuktepe and Kapçak (2013) improve the results in [6].

The purpose of this study is to investigate the effect of the immigration parameter $[5,10,11,13,14,15]$ on the dynamics of the model in [6] under the constant searching behavior as follows.

$$
\begin{aligned}
H_{t+1} & =\frac{R H_{t}}{\left(1+H_{t}\right)^{b}} e^{-P_{t}}+\beta ; \quad R, b, \beta>0 \\
P_{t+1} & =H_{t}\left(1-e^{-P_{t}}\right)
\end{aligned}
$$

where, $H_{t}$ is the population size at time $t, R$ is the intrinsic growth rate, $\beta$ is immigration parameter which provide control of the population. Also, it is seen that the growth of the host are governed by the Hassel law [3]. "Hassell et al. [3] collected $R$ and $b$ values for about two dozen species from field and laboratory observations and noted that the majority of these cases were within the stable region". Here, the reason we took the constant searching behavior is to examine the effect of immigration on the parameter values $R$ and $b$. Because these parameters are bifurcation parameters. Stone and Hart (1999) investigated behaviors of some the general discrete-time single population model under immigration effect. It is seen that the general criteria which characterise the effect on population dynamics is unknow. However, some observation can be made on a single-humped nonnegative function. There are the assumption that the populations which is subject to sufficiently large effect of immigration will always simplify. In most model classes, the increase in some parameter values without immigration expose period-doubling biforcation leading to chaos. In such models, even a small migration effect can often adverse this behavior at last. 
Our study is about the immigration parameter on a two-species interacting system including the behavior of the host species exhibiting bifurcation with increasing parameter values. Altough the increase of the immigration effect lead to the disappearance of the population for some large parameter values, it has an impact on simplifying the system behavior. In addition to, this effect can save the population from extinction. As the immigration parameter passes the "one", a steady transition has been observed from one equilibrium point to another equilibrium point.

\section{Main result}

In this section, we investigate positive equilibrium points of the system (1); and analyze the stability of the system (1).

\subsection{Equilibrium point $\left(H^{*}, 0\right)$ of the system (1)}

Theorem 1. The system (1) has a unique positive equilibrium point $\left(H^{*}, 0\right)$ in case of

$$
\frac{-(\beta-b \beta-2)+\sqrt{\beta^{2}(1-b)^{2}+4(1+\beta)}}{2(b-2)}<H^{*}, \quad b>2 .
$$

Proof. We can obtain the equilibrium point of system (1) from equilibrium point definition as follows:

$$
\begin{aligned}
H^{*} & =\frac{R H^{*}}{\left(1+H^{*}\right)^{b}} e^{-P^{*}}+\beta \\
P^{*} & =H^{*}\left(1-e^{-P^{*}}\right) .
\end{aligned}
$$

Since $\beta>0, H^{*}$ isn't equal to zero. For $H^{*} \neq 0$ and $P^{*}=0$, we find $e^{-P^{*}}=1$. If the equilibrium point of system (1) exist, then it must be provided as follows:

$$
R=\frac{\left(1+H^{*}\right)^{b}\left(H^{*}-\beta\right)}{H^{*}}
$$

such that $H^{*}>\beta$. If we take $H^{*}=x$ in Eq.(3), then we can write the right side of Eq.(3) as the following the function

$$
F(x)=\frac{(1+x)^{b}(x-\beta)}{x} .
$$

We must show that $F$ is monotone. If the derivation of the function $F(x)$ is calculated, then we have

$$
F^{\prime}(x)=\frac{(1+x)^{b-1}}{x^{2}}\left[(b-2) x^{2}+(\beta-b \beta-2) x+\beta\right] .
$$

From there, the function of $F^{\prime}$ has roots

$$
x_{1}=\frac{-(\beta-b \beta-2)-\sqrt{\beta^{2}(1-b)^{2}+4(1+\beta)}}{2(b-2)}, \quad x_{2}=\frac{-(\beta-b \beta-2)+\sqrt{\beta^{2}(1-b)^{2}+4(1+\beta)}}{2(b-2)} .
$$

Here, $\beta-b \beta-2<0$ confirms for $b>2$. It is easily seen that $x_{1}<0$ and $x_{2}>0$. Now, if the necessary examinations are made, then we get the desired result.

Remark. The following statements are true for $F(x)$.

(i) If $b>2$, then $F(x) \rightarrow \infty$ as $x \rightarrow \infty$. 
(ii) If $b>2$, then $F(x) \rightarrow-\infty$ as $x \rightarrow 0^{+}$.

(iii) If $0<b<2$, then $F(x) \rightarrow 0$ as $x \rightarrow \infty$. There exist one critical point $x_{1}$ which is local maximum of function $F(x)$.

(iv) Let's define the following form

$$
f=\frac{R H^{*}}{H^{*}-\beta} \text { and } g=\left(1+H^{*}\right)^{b}
$$

from Eq.(3). $f$ is decreasing in $H^{*}$ when $H^{*}>\beta$ and $\lim _{H^{*} \rightarrow \beta^{+}} g=\infty$. $g$ is increasing in $H^{*}$ with $g(\beta)=(1+\beta)^{b}$.

\subsection{Equilibrium point $\left(H_{1}^{*}, P_{1}^{*}\right)$ of the system (1)}

Theorem 2. In case of

$$
0<\frac{\left(1+H_{1}^{*}\right)^{b}\left(H_{1}^{*}-\beta\right)}{R H_{1}^{*}}<1
$$

the system (1) has positive equilibrium point $\left(H_{1}^{*}, P_{1}^{*}\right)$ different from the positive equilibrium point $\left(H^{*}, 0\right)$.

Proof. If system (1) is taken into account for $H_{1}^{*} \neq 0$ and $P_{1}^{*} \neq 0$, by using equilibrium point definition, we obtain as follows.

$$
\begin{aligned}
H_{1}^{*}\left(1+H_{1}^{*}\right)^{b} & =R H_{1}^{*} e^{-P_{1}^{*}}+\beta\left(1+H_{1}^{*}\right)^{b} \Rightarrow R H_{1}^{*} e^{-P_{1}^{*}}=H_{1}^{*}\left(1+H_{1}^{*}\right)^{b}-\beta\left(1+H_{1}^{*}\right)^{b} \\
& \Rightarrow e^{-P_{1}^{*}}=\frac{H_{1}^{*}\left(1+H_{1}^{*}\right)^{b}-\beta\left(1+H_{1}^{*}\right)^{b}}{R H_{1}^{*}} . \\
& \Rightarrow P_{1}^{*}=-\ln \frac{\left(1+H_{1}^{*}\right)^{b}\left(H_{1}^{*}-\beta\right)}{R H_{1}^{*}} .
\end{aligned}
$$

such that $H_{1}^{*}>\beta$. If

$$
0<\frac{\left(1+H_{1}^{*}\right)^{b}\left(H_{1}^{*}-\beta\right)}{R H_{1}^{*}}<1,
$$

then $P_{1}^{*}>0$. If we combine Eq. (5) and the second equation of system (1), then we get

$$
P_{1}^{*}=H_{1}^{*}\left(1-\frac{\left(1+H_{1}^{*}\right)^{b}\left(H_{1}^{*}-\beta\right)}{R H_{1}^{*}}\right)
$$

If $P_{1}^{*}$ is written in the first equation in system (1), we get

$$
\begin{aligned}
H_{1}^{*} & =\frac{R H_{1}^{*}}{\left(1+H_{1}^{*}\right)^{b}} e^{-H_{1}^{*}\left(1-\frac{\left(1+H_{1}^{*}\right)^{b}\left(H_{1}^{*}-\beta\right)}{R H_{1}^{*}}\right)}+\beta \\
& \Rightarrow\left(1+H_{1}^{*}\right)^{b}\left(H_{1}^{*}-\beta\right)=R H_{1}^{*} e^{-H_{1}^{*}\left(1-\frac{\left(1+H_{1}^{*}\right)^{b}\left(H_{1}^{*}-\beta\right)}{R H_{1}^{*}}\right)} \\
& \Rightarrow R=\left(1+H_{1}^{*}\right)^{b}\left(1-\frac{\beta}{H_{1}^{*}}\right) e^{H_{1}^{*}\left(1-\frac{\left(1+H_{1}^{*}\right)^{b}\left(H_{1}^{*}-\beta\right)}{R H_{1}^{*}}\right)}
\end{aligned}
$$

Now, let's take $H_{1}^{*}=x$. Then we can write the last equality as

$$
G(x)=(1+x)^{b}\left(1-\frac{\beta}{x}\right) e^{x-\frac{x}{R}(1+x)^{b}\left(1-\frac{\beta}{x}\right)}
$$

Remark. Since $G(x) \rightarrow 0$ as $x \rightarrow \infty$, there exist one critical point which is local maximum of function $G$. Here, $G(\beta)=0$, $G^{\prime}(\beta)=\frac{(\beta+1)^{b}}{\beta} e^{\beta}>0$. 


\subsection{Stability analysis of system (1)}

In this section, we will examine the local stability of equilibrium points. Firstly, we assume that inequality (7) is not satisfied. Namely, we have only unique equilibrium point $\left(H^{*}, 0\right)$. Let's write (1) as

$$
\begin{gathered}
F\left(H_{t}, P_{t}\right)=\frac{R H_{t}}{\left(1+H_{t}\right)^{b}} e^{-P_{t}}+\beta \\
G\left(H_{t}, P_{t}\right)=H_{t}\left(1-e^{-P_{t}}\right) .
\end{gathered}
$$

If the Jacobian matrix of system (1) is created, then we have

$$
J=\left(\begin{array}{cc}
R(1+H)^{-b-1}[1+(1-b) H] e^{-P}-e^{-P} \frac{R H}{(1+H)^{b}} \\
\left(1-e^{-P}\right) & H e^{-P}
\end{array}\right) .
$$

The Jacobian matrix which is evaluated the neighborhood of $\left(H^{*}, 0\right)$ is written as

$$
J=\left(\begin{array}{cc}
R\left(1+H^{*}\right)^{-b-1}\left[1+(1-b) H^{*}\right]-\frac{R H^{*}}{\left(1+H^{*}\right)^{b}} \\
0
\end{array}\right) .
$$

From this, we get that $\lambda_{1}=R\left(1+H^{*}\right)^{-b-1}\left[1+(1-b) H^{*}\right]$ and $\lambda_{2}=H^{*}$. So, $\left(H^{*}, 0\right)$ is local stable if

$$
R\left(1+H^{*}\right)^{-b-1}\left[1+(1-b) H^{*}\right]<1 \text { and } H^{*}<1 .
$$

Let's assume that $R\left(1+H^{*}\right)^{-b-1}\left[1+(1-b) H^{*}\right]>1$ and $H^{*}>1$. So, $\left(H^{*}, 0\right)$ is unstable, and the system of (1) has uniqe equilibrium point $\left(H_{1}^{*}, P_{1}^{*}\right)$. Let's consider charecteristic equation of Jacobian matrix of system (1) for $\left(H_{1}^{*}, P_{1}^{*}\right)$. So, we get

$$
\lambda^{2}-t r J \lambda+\operatorname{det} J=0
$$

If (see [1], page 64)

$$
|t r J|<1+\operatorname{det} J<2
$$

then $\left(H_{1}^{*}, P_{1}^{*}\right)$ is stable such that

$$
\begin{gathered}
\operatorname{tr} J=R\left(1+H_{1}^{*}\right)^{-b-1}\left[1+(2-b) H_{1}^{*}\right] e^{-P_{1}^{*}} \\
\operatorname{det} J=R\left(1+H_{1}^{*}\right)^{-b-1}\left[1+(1-b) H_{1}^{*}\right] H_{1}^{*} e^{-2 P_{1}^{*}}+\left(1-e^{-P_{1}^{*}}\right) e^{-P_{1}^{*}} \frac{R H_{1}^{*}}{\left(1+H_{1}^{*}\right)^{b}} .
\end{gathered}
$$

As a result of this, we get that $\left(H_{1}^{*}, P_{1}^{*}\right)$ is local stable from (12) if

$$
\begin{aligned}
& e^{-P_{1}^{*}}\left[R\left(1+H_{1}^{*}\right)^{-b-1}\left(1+(2-b) H_{1}^{*}\right)-\left(1-e^{-P_{1}^{*}}\right) \frac{R H_{1}^{*}}{\left(1+H_{1}^{*}\right)^{b}}\right]-e^{-P_{1}^{*}} H_{1}^{*} R\left(1+H_{1}^{*}\right)^{-b-1}\left(1+(1-b) H_{1}^{*}\right)<1, \\
& e^{-P_{1}^{*}}\left[R\left(1+H_{1}^{*}\right)^{-b-1}\left(1+(2-b) H_{1}^{*}\right)-\left(1-e^{-P_{1}^{*}}\right) \frac{R H_{1}^{*}}{\left(1+H_{1}^{*}\right)^{b}}\right]+e^{-P_{1}^{*}} H_{1}^{*} R\left(1+H_{1}^{*}\right)^{-b-1}\left(1+(1-b) H_{1}^{*}\right)>-1,
\end{aligned}
$$

and

$$
R\left(1+H_{1}^{*}\right)^{-b-1}\left[1+(1-b) H_{1}^{*}\right] H_{1}^{*} e^{-P_{1}^{*}}+\left(1-e^{-P_{1}^{*}}\right) e^{-P_{1}^{*}} \frac{R H_{1}^{*}}{\left(1+H_{1}^{*}\right)^{b}}<1
$$




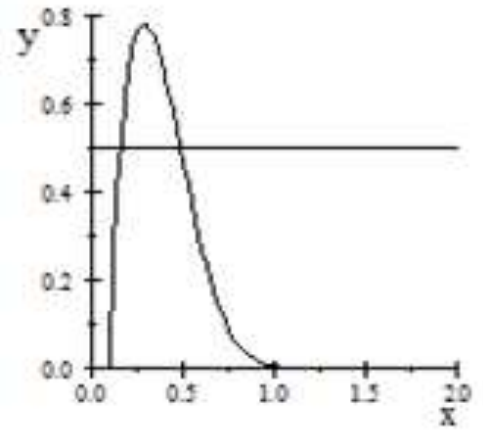

(a) Graph of the function of $y=G(x)$ and the line of $y=R$ such that $b=$ $2.1, \beta=0.1$ and $R=0.5$.

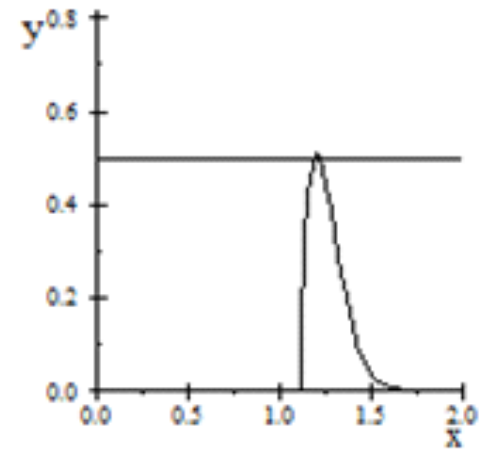

(b) Graph of the function of $y=G(x)$ and the line of $y=R$ such that $b=$ $2.1, \beta=1.1$ and $R=0.5$.

Fig. 1

Corollary 1. When the inequality (7) is not provided, $\left(H^{*}, 0\right)$ is uniqe positive equilibrium point of system (1) under the inequality $\frac{-(\beta-b \beta-2)+\sqrt{\beta^{2}(1-b)^{2}+4(1+\beta)}}{2(b-2)}<H^{*}, \quad b>2$. If the inequality (7) is provided such that $\frac{-(\beta-b \beta-2)+\sqrt{\beta^{2}(1-b)^{2}+4(1+\beta)}}{2(b-2)}<H^{*}, b>2$, the system (1) has the positive equilibrium points $\left(H^{*}, 0\right)$ and $\left(H_{1}^{*}, P_{1}^{*}\right)$.

Corollary 2. If the system (1) has the equilibrium points $\left(H^{*}, 0\right)$ and $\left(H_{1}^{*}, P_{1}^{*}\right)$, the following statements hold true.

(i) If the equilibrium point $\left(H^{*}, 0\right)$ is not stable, then the equilibrium point $\left(H_{1}^{*}, P_{1}^{*}\right)$ is stable under conditions (13),(2.3) and (15).

(ii) If the equilibrium point $\left(H_{1}^{*}, P_{1}^{*}\right)$ is not stable, then the equilibrium point $\left(H^{*}, 0\right)$ is stable under condition (11).

Also, the following examples confirm our the theoretical result.

\section{Numerical simulations}

Here, we confirm our theoretical results by using Mathematica and Scientific WorkPlace 5.5 program. The initial conditions are taken $H_{0}=0.2$ and $P_{0}=0.1$.

Example 1. Let's choose the system as

$$
\begin{aligned}
H_{t+1} & =\frac{0.5 H_{t}}{\left(1+H_{t}\right)^{2.1}} e^{-P_{t}}+\beta \\
P_{t+1} & =H_{t}\left(1-e^{-P_{t}}\right)
\end{aligned}
$$

where the functions $G(x)$ as follows.

$$
G(x)=(1+x)^{2.1}\left(1-\frac{\beta}{x}\right) e^{x\left(1-\frac{(1+x)^{2.1}(x-\beta)}{0.5 x}\right)} .
$$

Example 2. Let's choose the system as

$$
\begin{aligned}
H_{t+1} & =\operatorname{frac} 0 H_{t}\left(1+H_{t}\right)^{6} e^{-P_{t}}+\beta \\
P_{t+1} & =H_{t}\left(1-e^{-P_{t}}\right)
\end{aligned}
$$




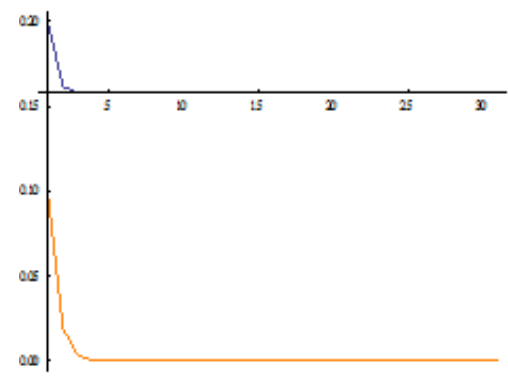

(a) Time series diagram of the model (1) where $b=2.1, \beta=0.1$ and $R=0.5$.

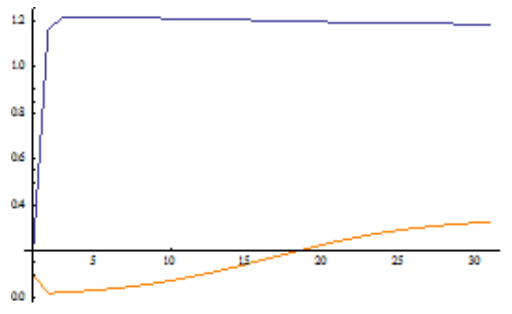

(b) Time series diagram of the model (1) where $b=2.1, \beta=1.1$ and $R=0.5$.

Fig. 2

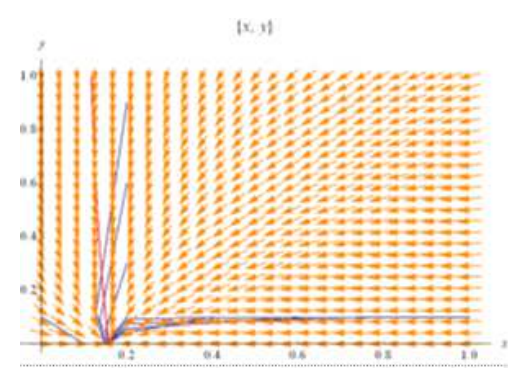

(a) Phase Diagram of the model (1) where $b=2.1, \beta=0.1$ and $R=0.5$.

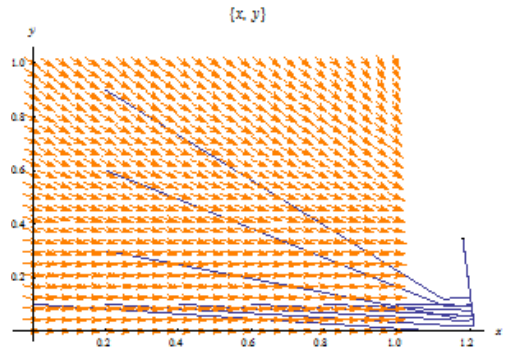

(b) Phase Diagram of the model (1) where $b=2.1, \beta=1.1$ and $R=0.5$.

Fig. 3

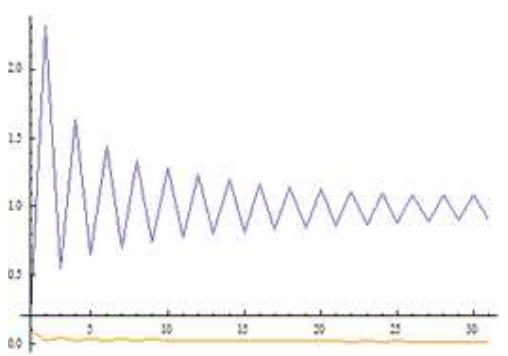

(a) Time series diagram of the model (1) where $b=6, \beta=0,5$ and $R=30$.

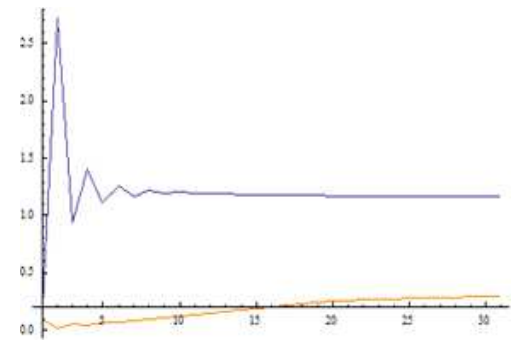

(b) Time series diagram of the model (1) where $b=6, \beta=0.9$ and $R=30$.

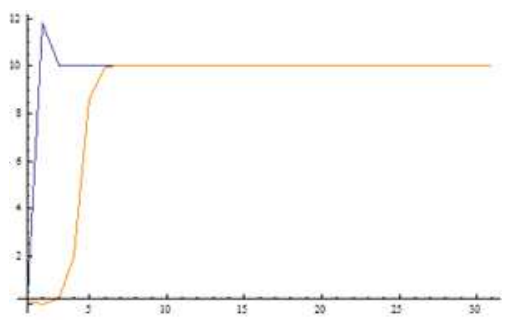

(c) Time series diagram of the model (1) where $b=6, \beta=10$ and $R=30$.

Fig. 4

where the functions $G(x)$ as follows

$$
G(x)=(1+x)^{6}\left(1-\frac{\beta}{x}\right) e^{x\left(1-\frac{(1+x)^{6}(x-\beta)}{30 x}\right)}
$$

\section{Discussion and conclusion}

Most studies dealing with the immigration effect on population models focus on the stability analysis of the equilibrium points. When a population is exposed to an invasion, it is important to determine under what conditions the population's 
equilibrium point will be stable or unstable. Even if the impact on a population is a small constant value, it leads to major behavioral changes on populations. That is why we are interested in exploring the consequences of immigration effect. Does this effect reach the population exhibiting chaos behavior or cyclical oscillations of long period to a balance by restoring equilibrium point? In this paper, we investigated the equilibrium points of discrete-time host-parasite model and examined the local stability of this system. So, the impact of the immigration parameter on the system is presented as mathematical consequences.which offer an effect that simplifies the system.

\section{Acknowledgements}

The authors would like to sincerely thank the referees for their careful reading of the manuscript and valuable suggestions.

\section{Competing interests}

The authors declare that they have no competing interests.

\section{Authors' contributions}

All authors have contributed to all parts of the article. All authors read and approved the final manuscript.

\section{References}

[1] L.J.S. Allen.: An introduction to mathematical biology, Pearson, New Jersey, (2007).

[2] J.R. Beddington, C. A. Free and J. H. Lawton.: Dynamic complexity in predator-prey models framed in difference equation, Nature 255 (1975), 58-60.

[3] M.P. Hassell, H.N. Comins.: Discrete-time models for two species competition. Theor. Popul. Biol. 9 (1976), 202-221.

[4] S. Kapçak, U. Ufuktepe and S. Elaydi.: Stability and invariant manifolds of a generalized Beddington host-parasitoid model, Journal of Biological Dynamics 7 (2013), 233-253.

[5] M.R.S Kulenovic, G. Ladas.: Dynamics of second order rational difference equations: with open problems and conjectures, America, (2002).

[6] J.C. Misra, A. Mitra.: Instabilities in single-species and host-parasite systems:period-doubling bifurcations and chaos, Comput. Math. Appl. 52 (2006),525-538.

[7] J. D. Murray.: Mathematical Biology, New York , (2002).

[8] A. Nicholson and V. Bailey.: The balance of animal population, 3, Proc. Zool. Soc. Lond., (1935).

[9] U. Ufuktepe, S. Kapçak.: Stability analysis of a host parasite model, Advances of Difference Equations 79 (2013) 2-7.

[10] Ö. Ak Gümüş, F. Kangalgil.: Allee effect and stability in discrete-time host-parasitoid model, Journal of Advanced Research in Applied Mathematics, 7 (2015), 94-99.

[11] Ö. Ak Gümüş.: Dynamical conseqences and stability analysis of a new host parasitoid model, General Mathematics Notes 27(2015), 9-15.

[12] Q. Din, Ö. Ak Gümüş and H. Khalil.: Neimark-Sacker bifurcation and chaotic behavior of a modified host-parasitoid model, Zeitschrift fur Naturforschung, 72 (2017), 25-37.

[13] L Stone, D. Hart, Effects of immigration on the dynamics of simple population models: Theoretical Population Biology 55 (1999), 227-234.

[14] H.I. McCallum.: Effects of immigration on chaotic population dynamics, Journal Theoretical Biology, 154 (1992) $277-284$.

[15] R. D. Holt.: Immigration and the dynamics of peripheral populations, in "Advances in Herpetology and Evolutionary Biology", Museum of Comparative Zoology, Harvard University,Cambridge, MA, (1983). 